\title{
Gestión de la continuidad del servicio ante emergencias y desastres en salud: enfoque para la reactivación productiva
}

\section{Service Continuity Management in Health Emergencies and Disasters: Approach for Productive Reactivation}

Gestão da continuidade do serviço en emergência e desastres na saúde: enfoque para a reativação produtiva

\author{
Catalina Artavia Pereira \\ Universidad de Costa Rica. San José, Costa Rica \\ catalina.artavia@ucr.ac.cr \\ https://orcid.org/0000-0001-6745-3934 \\ Felipe Calvo Villalobos \\ Universidad de Costa Rica. San José, Costa Rica \\ fcalvo@inteco.org \\ https: https://orcid.org/0000-0001-7490-6268
}

DOI: https://doi.org/10.32719/25506641.2021.10.6

Recibido: 15 de octubre de 2020 - Revisado: 20 de noviembre de 2020

Aceptado: 26 de enero de 2021 - Publicado: 1 de julio de 2021

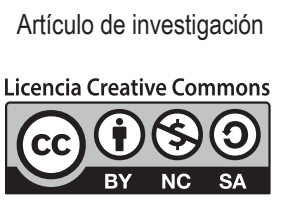




\section{Resumen}

En el presente artículo se describe el proceso de elaboración y contenido del documento normativo de protocolos sectoriales para la reactivación de los sectores productivos en el caso de Costa Rica. Tiene como principal objetivo presentar la forma en que las medidas gubernamentales evolucionan para dar respuesta a situaciones de emergencia sanitaria por medio de protocolos sectoriales para la continuidad de los servicios y de los sectores productivos. Se argumenta que la continuidad de las operaciones en las organizaciones públicas y privadas, es aún más relevante y prioritario en el contexto actual ante la presencia de múltiples amenazas que influyen en la estabilidad del sistema económico, social y político. La metodología empleada para el desarrollo de los protocolos sectoriales se basó en la revisión bibliográfica y en las buenas prácticas de otros países en su elaboración, así como las recomendaciones de las autoridades sobre lineamientos sanitarios para la COVID-19. En conclusión, se reconoce la importancia de los instrumentos de política pública emitidos ante una situación de emergencia y desastres en salud.

Palabras clave: continuidad del negocio, resiliencia, protocolos sectoriales, reactivación económica, COVID-19.

JEL: I1 Política pública, regulación, sanidad pública.

\section{Abstract}

This article describes the elaboration and content process of the normative document of sectorial protocols for productive sector reactivation in the case of Costa Rica. Its main objective is to present how the government measures evolve to respond to health emergencies through sectorial protocols for service continuity and productive sectors. It is argued that the continuity of operations in public and private organizations is even more relevant and a priority in the event of multiple threats influencing the stability of the economic, social and political system. The methodology used for the sectorial protocol development was based on the bibliographic review and other countries experiences in the protocol elaboration, and the recommendations through the health guidelines for COVID-19. In conclusion, the importance of public policy instruments issued in the face of emergencies and health disasters.

Keywords: business continuity, resilience, sector protocols, economic reactivation, COVID-19. JEL: I1 Analysis of health care markets.

\section{Resumo}

O presente artigo descreve o processo de elaboração e o conteúdo do documento normativo de protocolos setoriais para a reativação dos setores produtivos para o caso da Costa Rica. Tem como principal objetivo apresentar a forma na qual as medidas governamentais evoluem para responder a situações de emergência sanitária por meio de protocolos setoriais para a continuidade dos serviços e dos setores produtivos. Argumenta-se que a continuidade das operações nas organizações públicas e privadas é ainda mais relevante e prioritária no atual contexto devido à presença de múltiplas ameaças que influem sobre 
a estabilidade do sistema econômico, social e político. A metodologia empregada para o desenvolvimento dos protocolos setoriais se baseou na revisão bibliográfica e em boas práticas de outros países na elaboração de protocolos, assim como nas recomendações das autoridades mediante os regulamentos sanitários emitidos por conta do Covid-19. Em conclusão, identifica-se a importância dos instrumentos de política pública emitidos frente a uma situação de emergências e desastres de saúde.

Palavras-chave: continuidade do negócio, resiliência, protocolos setoriais, reativação econômica, COVID-19.

JEL: I11 Políticas públicas, regulamento, sanidade pública.

\section{Introducción}

lo largo de la historia, la humanidad ha tenido que enfrentar y lidiar
con enfermedades que han afectado a la población mundial, ejemplo
de ello es la muerte negra (peste) que llegó a Europa en 1334 y aniquiló en seis años a 20 millones de habitantes; las siete pandemias del cólera (1816 y 1991), enfermedad que aún hoy sigue cobrando vidas en muchos países de Asia y América Latina; las epidemias de tifo que diezmaron a los combatientes en las guerras europeas de finales del siglo XV y principios del XVI; y, la epidemia más reciente, que marcó el cambio hacia el siglo XXI, el síndrome respiratorio agudo grave (SARS, por sus siglas en inglés de severe acute respiratory syndrome) (Gala 2005).

Destaca adicionalmente, en el siglo XX, la pandemia de influenza de 1918-1919 (virus H1N1), la cual ocasionó alrededor de 50 millones de fallecimientos a nivel mundial, la cual es considerada como la influenza más mortal en la historia moderna (Centros para el Control y la Prevención de Enfermedades 2019).

Recientemente, en diciembre de 2019, surgió un virus desconocido que fue registrado en la ciudad de Wuhan (China), que provoca una enfermedad infecciosa causada por el coronavirus. Actualmente, es reconocido como la COVID-19 y fue declarada por la Organización Mundial de la Salud (OMS) como pandemia (OMS 2020).

En el marco de la declaratoria de la pandemia por la COVID-19, y de acuerdo con el Reglamento Sanitario Internacional (OPS 2005), las diferentes organizaciones competentes en salud y manejo de emergencias a nivel 
mundial, basados en el procedimiento establecido, ejecutan acciones con el fin de manejar la preparación y respuesta ante la pandemia. En ese sentido, el Reglamento Sanitario Internacional tiene como fin prevenir la propagación internacional de enfermedades, controlarla y dar una respuesta de salud pública proporcionada y restringida a los riesgos para la salud pública y evitar al mismo tiempo las interferencias innecesarias con el tráfico y el comercio internacionales (OMS 2005), en el mismo Reglamento se establece el procedimiento a seguir por parte de la OMS y los Estados miembros ante un evento declarado como emergencia de salud pública de importancia internacional.

Los países desarrollaron una serie de estrategias con el fin de contener el brote; entre las medidas adoptadas destacan: aislamiento domiciliario, cuarentena de casos sospechosos, distanciamiento social de pacientes de riesgo por tener una enfermedad grave, higiene sanitaria y dejar a la población actuar libremente (Gonzales et al. 2020). Otra de las estrategias implementadas por distintos países son las medidas de supresión las cuales corresponden a un mecanismo adaptativo que consiste en revertir el crecimiento del número de infectados reduciendo el número de casos a niveles bajos (Gonzales et al. 2020).

Por ejemplo, en el caso de Perú, la aplicación de la estrategia de supresión - como política pública - ha permitido reducir la curva de infectados en más del $50 \%$, pero no ha sido suficiente para mitigar el colapso del sistema sanitario. Dicha medida genera altos costos sociales por la existencia previa de características estructurales, fallas del Estado y una sociedad civil débil en su capacidad de reaccionar frente a la ineficiencia del Estado (Gonzales et al. 2020).

Precisamente, los efectos de una epidemia o pandemia afectan la estabilidad y crecimiento económico de los países, como ejemplo, el costo estimado anual de la influenza pandémica por AH1N1 fue aproximadamente de USD 500.000 millones (que corresponde al 0,6\% del ingreso mundial), incluida la pérdida de ingresos y el costo intrínseco del aumento en la mortalidad. Al afectar negativamente el comercio y desalentar la inversión, estos eventos reducen el crecimiento del producto interno bruto de un país (OPS 2019).

América se caracteriza por estar expuesta a amenazas, por ejemplo, el territorio experimentó más de 2000 desastres de gran magnitud entre 1970 a 2015, sucesos que afectaron a más de 250 millones de personas. Los de- 
sastres severos más recientes en la región de América Latina y el Caribe incluyen el terremoto de Haití en 2010 (que causó pérdidas económicas por USD 7800 millones y 300.000 muertes); el terremoto de Chile en 2010 (USD 30.000 millones en pérdidas económicas y 500 muertes); las inundaciones de Colombia en 2010-2011 (que causaron pérdidas económicas de USD 5000 millones y 400 muertes), y el terremoto en Ecuador en 2016 (que causó USD 3300 millones en pérdidas económicas y 660 muertes) (Centro de Investigación sobre la Epidemiología de los Desastres 2017).

Desde el punto de vista de los efectos sobre la salud pública de los desastres, estos pueden reflejarse en los siguientes niveles: mortalidad, enfermedades transmisibles, capacidad del sistema de servicios sanitarios, efectos en la salud mental y afectación en el desarrollo (González, Castro y del Busto 2002).

En ese sentido, es clave la adecuada articulación de los sistemas de gestión del riesgo, como un mecanismo que permita una oportuna coordinación, movilización de estructuras, procedimientos y recursos e incorpore la gestión del riesgo como un eje transversal en la planificación y funcionamiento del sector público y privado.

De este modo, es necesaria una correcta articulación entre los sistemas de gestión del riesgo de desastres de los países y el sistema de salud. La emergencia por la COVID-19 puso de manifiesto un riesgo de tipo biológico, para el cual es preciso considerar mecanismos o instrumentos de gestión que permitan atender las emergencias con características epidemiológicas.

Desde un enfoque multiamenaza se integran los siguientes elementos: "1. la selección de múltiples amenazas importantes que afronta el país, y 2. los contextos particulares en los que pueden producirse sucesos peligrosos simultáneamente, en cascada o de forma acumulativa a lo largo del tiempo, y teniendo en cuenta los posibles efectos relacionados entre sí” (ONU 2016, 18).

Para el caso de Costa Rica, en el contexto multiamenaza en el que se desenvuelve, es necesario un enfoque que tome en cuenta dichos elementos. Las emergencias en salud como la ocasionada por la COVID-19, generan una serie de consecuencias sociales, técnicas, científicas, económicas, ambientales, éticas y políticas (PS 2015).

Dichas consecuencias se reflejan en costos para el sistema de salud — público y privado-, del tratamiento médico de los infectados y del control de 
los brotes; las epidemias causan ausentismo laboral de los enfermos y sus cuidadores disminuyen su eficacia en el trabajo, lo cual merma la productividad. Aunado a lo anterior, se genera un temor a la infección que puede originar aislamiento social o cierre de escuelas, empresas, comercios, transporte y servicios públicos, lo que perturba las actividades económicas y sociales (Bloom, Cadarette y Sevilla 2018).

Las epidemias, por su naturaleza, cuentan con causas que complican su prevención y control, como la "rápida transmisión de las enfermedades, a nivel nacional e internacional, la posibilidad de epidemias se ve magnificada no solo por la globalización, sino por el doble fenómeno del cambio climático y la urbanización; el cambio climático está expandiendo el hábitat de diversos vectores de enfermedades comunes" (Bloom, Cadarette y Sevilla 2018, 47).

En ese sentido, es necesaria la aplicación de medidas para minimizar el efecto sanitario del brote para lo cual surge la elaboración de lineamientos y protocolos estandarizados que permitan la apertura paulatina del comercio, de los diferentes sectores económicos y de las actividades.

En la primera parte del artículo se contextualiza de la situación de emergencia y la forma de abordaje a nivel sectorial en Costa Rica; la segunda parte describe la respuesta sectorial para la continuidad de los sectores durante la emergencia por la COVID-19; la tercera presenta los elementos y la importancia de la continuidad del negocio y resiliencia en el contexto actual, $\mathrm{y}$, por último, se exponen las conclusiones del estudio.

\section{Materiales y métodos}

La metodología corresponde a la utilizada para el desarrollo de los protocolos sectoriales, el cual es el principal insumo del presente artículo.

En cuanto al enfoque, comprende el tipo cualitativo en tanto se describen los elementos básicos de los protocolos sectoriales para la reactivación de los sectores productivos, la importancia de instrumentos estandarizados y la adaptación de la política pública a los diferentes tipos de eventos como las emergencias sanitarias. 
Su tipología es descriptiva, al especificar características del fenómeno analizado, y exploratoria debido a que existe un cierto vacío de conocimiento, porque aún no hay un registro documental robusto atinente a la continuidad de los servicios ante situaciones de emergencias sanitarias del alcance de la pandemia ocasionada por la COVID-19.

Se realizó una revisión bibliográfica de fuentes secundarias con documentos oficiales suministrados por el Ministerio de Salud, Ministerio de la Presidencia, Comisión Nacional de Prevención de Riesgos y Atención de Emergencias, todas entidades de Costa Rica, y documentación sobre las buenas prácticas implementadas por otros países en la elaboración de protocolos, así como recomendaciones de las autoridades, por medio de los lineamientos sanitarios por la COVID-19.

\section{Organización para la atención de la emergencia por la COVID-19: caso Costa Rica}

El 6 de marzo de 2020, se presentó el primer caso de la COVID-19 en Costa Rica; a raíz de ello, el Ministerio de Salud de Costa Rica, como ente rector en salud, y las diferentes instancias a nivel nacional iniciaron la coordinación para la atención de la emergencia.

El Ministerio de Salud es el organismo que ejerce la rectoría dentro de dicho sector para un abordaje efectivo de las emergencias sanitarias o desastres y responde a la necesidad de articular las acciones institucionales e interinstitucionales (CR Ministerio de Salud 2019).

Bajo el amparo de dicha normativa, el Ministerio de Salud y el Viceministerio de la Presidencia elaboran la Directriz No. 082-MP-S "Sobre los protocolos para la reactivación y continuidad de los sectores durante el estado de emergencia nacional por COVID-19" (CR 2020), la cual tiene como objetivo instruir a las autoridades de la administración pública central e instar a las autoridades de la administración pública descentralizada, a iniciar un proceso coordinado y participativo con el sector privado para la aplicación de medidas de prevención y mitigación de la COVID-19, que permitan la reactivación y continuidad de los centros de trabajo, actividades y servicios, según el comportamiento epidemiológico de dicha enfermedad (CR 2020). 
Para el cumplimiento del objetivo de la Directriz, se indica el siguiente procedimiento:

- Cada autoridad debe iniciar un proceso coordinado y participativo con su respectivo sector o sectores, según el tipo de centro de trabajo, actividad o servicio.

- El Ministerio de Salud emitirá los lineamientos generales que deben contemplar los protocolos específicos por sector.

- Todos los protocolos específicos deberán apegarse a los lineamientos generales del Ministerio de Salud.

- Todos los sectores podrán remitir propuestas de protocolos específicos a los ministerios o presidencias ejecutivas, según corresponda (CR Presidencia 2020, art. 4).

Una vez habilitada la actividad, servicio o centro de trabajo, el Ministerio de Salud realizará inspecciones aleatorias para la verificación del cumplimiento de los protocolos específicos, así como de los lineamientos generales emitidos por el Ministerio de Salud por el COVID-19 (CR 2020, art. 5). En cuanto al cumplimiento, las autoridades deben informar al Consejo de Gobierno el seguimiento del procedimiento anterior.

Tabla 1

Actores y funciones de protocolos para la reactivación y continuidad de los sectores durante el estado de emergencia nacional por la COVID-19

\begin{tabular}{|l|l|l|}
\hline \multicolumn{1}{|c|}{ Actores } & \multicolumn{1}{|c|}{ Funciones } & \multicolumn{1}{c|}{ Roles } \\
\hline $\begin{array}{l}\text { Ministerio } \\
\text { de la Presidencia }\end{array}$ & $\begin{array}{l}\text { Coordinar y apoyar las acciones de } \\
\text { las instituciones para la concreción } \\
\text { de los objetivos de la directriz. }\end{array}$ & Coordinador y articulador \\
\hline Consejo de Gobierno & $\begin{array}{l}\text { Cada persona jerarca deberá infor- } \\
\text { mar al Consejo de Gobierno sobre } \\
\text { el seguimiento del proceso seña- } \\
\text { lado. }\end{array}$ & Seguimiento \\
\hline $\begin{array}{l}\text { Secretaría del Consejo } \\
\text { de Gobierno }\end{array}$ & Registrar el protocolo remitido. & $\begin{array}{l}\text { Sistematización-repositorio } \\
\text { de protocolos }\end{array}$ \\
\hline
\end{tabular}




\begin{tabular}{|l|l|l|}
\hline \multicolumn{1}{|c|}{ Actores } & \multicolumn{1}{|c|}{ Funciones } & \multicolumn{1}{c|}{ Roles } \\
\hline Ministerio de Salud & $\begin{array}{l}\text { Emitir los lineamientos generales } \\
\text { que deben contemplar los protoco- } \\
\text { los específicos por sector. }\end{array}$ & $\begin{array}{l}\text { Emisor, verificador-evaluador } \\
\text { y rector en salud }\end{array}$ \\
\hline $\begin{array}{l}\text { Autoridades } \\
\text { de la Administración } \\
\text { pública central } \\
\text { y administración pública } \\
\text { descentralizada }\end{array}$ & $\begin{array}{l}\text { Iniciar un proceso coordinado se- } \\
\text { gún su ámbito de competencia, } \\
\text { para la generación de protocolos }\end{array}$ & $\begin{array}{l}\text { Cocordinador } \\
\text { Acompañamiento } \\
\text { Aprobación } \\
\text { Seguimiento }\end{array}$ \\
\hline $\begin{array}{l}\text { Sector o subsector } \\
\text { Generación de protocolos especi- } \\
\text { ficos. }\end{array}$ & $\begin{array}{l}\text { Ejecutor } \\
\text { Comunicador }\end{array}$ \\
\hline actividad o servicio & $\begin{array}{l}\text { Implementar los protocolos espe- } \\
\text { cíficos. }\end{array}$ & Implementación \\
\hline
\end{tabular}

Fuente: adaptado de la Directriz No. 082-MP-S (CR Presidencia 2020).

El proceso de implementación de la Directriz se presenta en la figura 1 donde se pueden visualizar un total de cuatro niveles de actuación encabezado por el Consejo de Gobierno, seguido de los ministerios según el sector correspondiente, el Ministerio de Salud como ente rector y el Ministerio de la Presidencia, las organizaciones que conforman los sectores y por último los locales y comercios a nivel nacional.

El Consejo de Gobierno cuenta con el rol de repositorio de los diferentes protocolos sectoriales; el Ministerio de la Presidencia es el encargado de la articulación y facilitación del proceso, y genera una serie de herramientas guía dirigidas a los sectores. Los ministerios, según sus ámbitos, cumplen con la función de acompañamiento, aprobación, seguimiento, desarrollo y comunicación de los protocolos que llegan a implementar los centros de trabajo, actividades o servicios. 
Figura 1

\section{Esquema de funcionamiento para implementación de protocolos sectoriales}

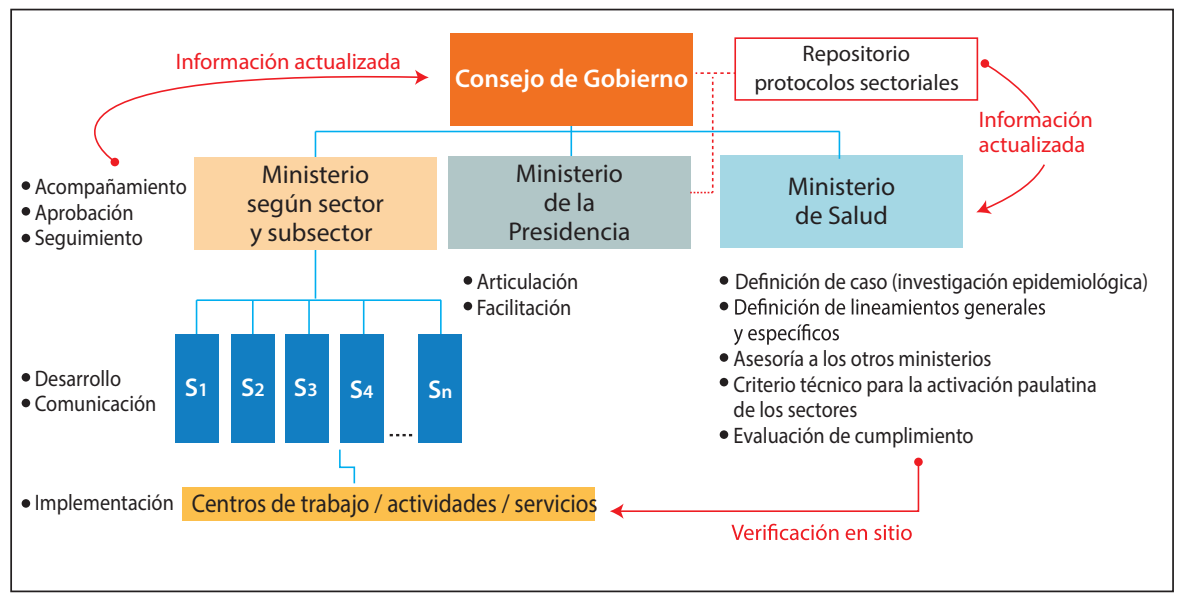

Fuente: Ministerio de la Presidencia, Ministerio de Salud, Universidad de Costa Rica INTECO (2020).

De esta forma, fue elaborado un flujo de desarrollo, aprobación y liberación de protocolos sectoriales, con el fin de generar una mejor comprensión en los sectores y subsectores. Se puede identificar en un total de cinco pasos:

Paso I: conocer el contenido de la Directriz No. 082-MP-S y los lineamientos de salud.

Paso II: desarrollo del protocolo sectorial tomando como base los "Requisitos para la elaboración de protocolos sectoriales para la implementación de Directrices y Lineamientos sanitarios para COVID-19”, coordinando con el representante de cada sector.

Paso III: aprobación del protocolo desarrollado, sometiéndolo a la valoración del ministerio correspondiente.

Paso IV: una vez aprobado el protocolo, se procede con la difusión de la información a los sectores para que utilicen el protocolo como insumo para su implementación.

Paso V: el Ministerio de Salud procede a realizar una evaluación aleatoria para verificar el cumplimiento con respecto al protocolo. 
Figura 2

Flujo de desarrollo, aprobación y liberación de protocolo sectorial

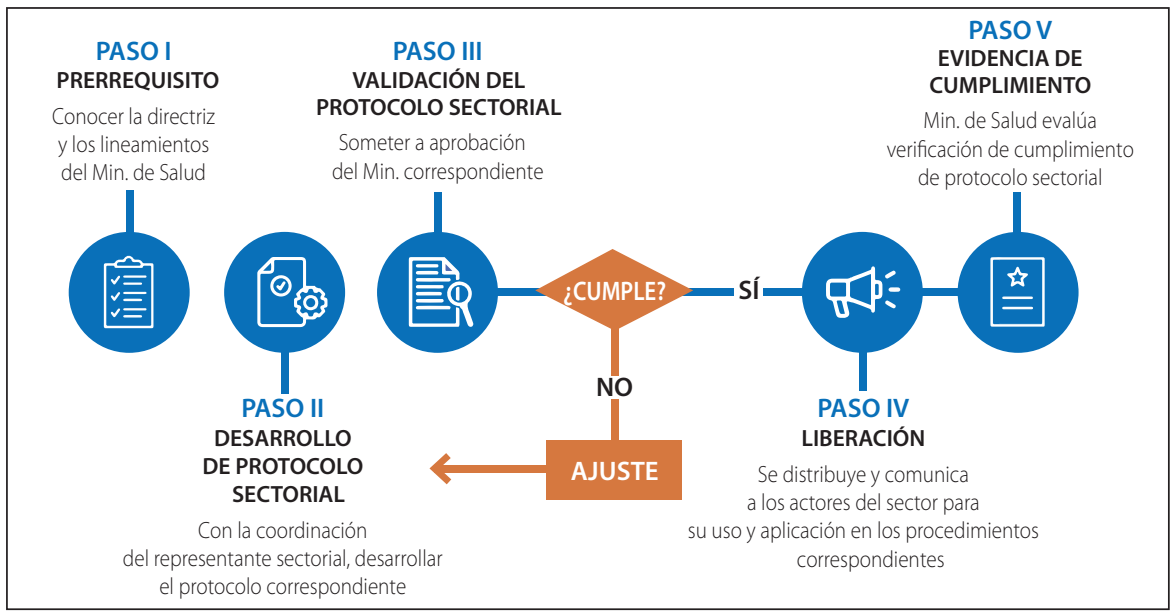

Fuente: Ministerio de la Presidencia, Ministerio de Salud, Universidad de Costa Rica INTECO (2020).

\section{Respuesta sectorial para la continuidad de los sectores durante la emergencia por la COVID-19: protocolos para el cumplimiento de lineamientos sanitarios}

En el marco de la Directriz No. 082-MP-S, se elaboró una caja de herramientas con instrumentos que permiten una mejor comprensión, aplicación y seguimiento de la misma. La caja de herramientas contiene un total de cinco instrumentos:

1. Documento "INTE/ DN-MP-S-19:2020 Requisitos para la elaboración de protocolos sectoriales para la implementación de directrices y lineamientos sanitarios para la COVID-19".

2. Anexo tipo plantilla para elaborar los protocolos sectoriales.

3. Lista de comprobación de protocolos sectoriales para la implementación de directrices y lineamientos sanitarios para la COVID-19.

4. Lista de sectores para la aplicación de los protocolos.

5. Video del proceso de elaboración e implementación de las diferentes herramientas mencionadas. 
Los requisitos para la elaboración de protocolos sectoriales se encuentran divididos en diez apartados los cuales se detallan en la tabla 2.

Tabla 2

Contenido del documento: requisitos para la elaboración de protocolos sectoriales para la implementación de directrices y lineamientos sanitarios para la COVID-19

\begin{tabular}{|l|l|}
\hline \multicolumn{1}{|c|}{ Contenido } & \multicolumn{1}{|c|}{ Descripción } \\
\hline Objetivo y campo de aplicación & $\begin{array}{l}\text { Define el tema y la finalidad del documento nor- } \\
\text { mativo, así como sus límites. }\end{array}$ \\
\hline Documentos y lineamientos de referencia & $\begin{array}{l}\text { Listado de lineamientos sanitarios vinculantes } \\
\text { al sector o subsector para el correcto funciona- } \\
\text { miento. }\end{array}$ \\
\hline Definiciones y abreviaturas & $\begin{array}{l}\text { Conceptos que el sector o subsector considere } \\
\text { pertinente. }\end{array}$ \\
\hline Principios & Principios considerados como buenas prácticas. \\
\hline Prerrequisitos de implementación & $\begin{array}{l}\text { Compromiso de cumplir con la Directriz 0-82 y } \\
\text { con los lineamientos aplicables del Ministerio de } \\
\text { Salud. }\end{array}$ \\
\hline Higiene y desinfección & $\begin{array}{l}\text { Medidas de higiene y desinfección del lugar de } \\
\text { trabajo. }\end{array}$ \\
\hline Logística en el centro de trabajo & $\begin{array}{l}\text { Procedimientos en cuanto a plan de continuidad } \\
\text { del negocio, los turnos y horarios de trabajo. }\end{array}$ \\
\hline Actuación ante casos confirmados de las perso- & $\begin{array}{l}\text { Acciones a seguir si existe confirmación médica } \\
\text { de personas contagiadas dentro de las instala- } \\
\text { ciones de la organización. }\end{array}$ \\
\hline Comunicación & $\begin{array}{l}\text { Mecanismos de comunicación para compartir } \\
\text { información relacionada con la COVID-19 pro- } \\
\text { veniente del Ministerio de Salud. }\end{array}$ \\
\hline Aprobación, seguimiento y evaluación & $\begin{array}{l}\text { Declaración del ministro rector o autoridad de } \\
\text { acuerdo con la aprobación. }\end{array}$ \\
\hline Verificación del cumplimiento del protocolo & $\begin{array}{l}\text { El Ministerio de Salud debe realizar inspeccio- } \\
\text { nes aleatorias para la verificación del cumpli- } \\
\text { miento. }\end{array}$ \\
\hline
\end{tabular}

Fuente: adaptado de Ministerio de la Presidencia, Ministerio de Salud, Universidad de Costa Rica, INTECO (2020). 


\section{Importancia de la estandarización de los protocolos sectoriales}

Es difícil llegar a pensar que, ante una situación de pandemia, como en cualquier otro efecto adverso sobre la sociedad, se espere salir de ello a través de soluciones creativas sin la base clara de lo más importante y crítico en el momento. Es necesario que exista una combinación entre la necesidad de la generación de instrumentos normativos y de política pública de forma rápida que responda a la problemática que se pretende resolver, y una base sólida y rigurosa que sustente las decisiones basadas en modelos consolidados y de buenas prácticas de contenido y forma.

La estandarización se convierte en una herramienta clave para orientar el cumplimiento de las disposiciones, el control y seguimiento efectivo de las acciones y la uniformidad en cuanto a requerimientos solicitados por parte de las autoridades. La estandarización puede ser definida como la actividad de establecer, frente a problemas reales o potenciales, disposiciones para uso común y repetido, encaminadas a la obtención del grado óptimo de orden en un contexto dado (INTECO 2016). La estandarización es vital para garantizar que el producto final sea conciso; debe reconocerse no como una herramienta inflexible para disponer la forma como se debe hacer el trabajo, sino como una herramienta de respaldo para guiar la jornada laboral actual y para plasmar los avances que vayan surgiendo, tras la revisión y actualización de la manera que se realiza mejor, día con día (Yunuen 2016).

Desde un enfoque de procedimientos, se establece que la estandarización de procesos consiste en definir y uniformar procedimientos, de modo que todas las personas que participan en él aplican permanentemente los mismos procedimientos (Harrington 1994).

La importancia de la estandarización de los protocolos sectoriales consiste en su utilidad, porque la forma en que los sectores presentan sus protocolos, bajo un mismo formato y lógica secuencial, permite a las autoridades sanitarias realizar un mejor control, trazabilidad y evaluación del cumplimiento de cada uno de los apartados que debe contener los protocolos. De esta forma se evita el mal uso de información, la pérdida de datos relevantes, la duplicidad de información y la pérdida de tiempo en la corrección de elementos poco relevantes. 
Por medio de la estandarización de los protocolos sectoriales, se promueve una reapertura de actividades y prestación de productos y servicios de forma más oportuna, eficaz y eficiente. El hecho de contar con un protocolo guía que integre los elementos básicos para la higiene y desinfección de los espacios en los lugares de trabajo, la logística en el desarrollo de actividades, la forma de comunicación y de actuación ante casos sospechosos o confirmados por la COVID-19, contribuyen a una mejor coordinación, atención y control de la emergencia sanitaria.

Una herramienta estandarizada permite que, tanto las organizaciones que implementan la norma como los que la evalúan, tengan un criterio unificado y de esta manera se reduce la duplicación de criterios, la confrontación, así como resultados no esperados. Su fundamento se basa en el desempeño, por ello brinda la posibilidad de que independientemente de los recursos con los que se posean, la organización pueda demostrar su cumplimiento según sus capacidades de conformidad con los requisitos dados.

\section{Continuidad del negocio y resiliencia}

Producto de un evento de desastre o disruptivo, provocado por amenazas naturales o antrópicas, las organizaciones se ven afectadas y en ocasiones obligadas a pausar su actividad; sin embargo, deben contar con una estrategia que les permita continuar operando pese a la afectación ocurrida.

En ese sentido la continuidad del negocio o las operaciones toma importancia, teniendo como objetivo la sobrevivencia de las organizaciones durante el desastre (SELA 2013).

Dentro de dichas estrategias a desarrollar por las organizaciones, se encuentran los protocolos o planes de continuidad del negocio o de las operaciones, los cuales se deben desarrollar tomando en cuenta los diversos riesgos a los que están expuestos en un contexto multiamenaza, que no necesariamente se tiene certeza de cuándo o en qué momento puedan ocurrir, pero se deben establecer medidas preventivas para evitar que causen un impacto grande en la organización y que aseguren la continuidad de las funciones.

La continuidad del negocio o las operaciones se define de la siguiente forma: capacidad de la organización para continuar suministrando productos 
o servicios a niveles predefinidos aceptables, posterior a un incidente disruptivo (INTECO 2015).

Este concepto toma mayor importancia en las agendas de las organizaciones. A lo largo de los años, se asociaba a la forma en la que se recuperaban las actividades en el área de informática ante eventuales caídas en los sistemas de información. Posteriormente, se consideraron otros elementos que podían afectar el funcionamiento como infraestructura, estado del personal, contexto económico y social del país entre otros elementos del ambiente, que replantearon la forma en que las organizaciones gestionan sus riesgos.

El riesgo se define como la probabilidad y gravedad de accidentes o pérdidas ante la exposición a diversos peligros, incluyendo lesiones a las personas y la pérdida de recursos (Robles 2020). En ese sentido, es necesario conocer, entender y analizar las condiciones de riesgo existentes, así como los procesos que contribuyen a su configuración (Robles 2020).

Se contaba con la idea de que un evento adverso alcanza la categoría de desastre cuando ocasiona masivamente la muerte de personas y la destrucción de la propiedad. La experiencia, sin embargo, ha mostrado que un factor crucial, antes y después del impacto, resulta ser la organización de las instituciones y de la comunidad; este elemento es tan susceptible a sufrir daños como lo son la vida y los bienes, con un agravante, cuando se afecta la organización - y los mecanismos de protección y asistencia - la respuesta a las víctimas y al entorno es tardía y deficiente, por lo tanto, los efectos del impacto resultan más dañinos y duraderos, eso los hace mucho más costosos (Morales et al. 2008).

En el contexto actual, la pandemia ocasionada por la COVID-19 corresponde a un incidente disruptivo que ha implicado la paralización de las operaciones de los sectores a nivel mundial. Se ha puesto de manifiesto que no todas las organizaciones cuentan con estrategias para continuar brindando sus servicios o productos, es decir, acordes a un evento de esta naturaleza. Para el caso de Costa Rica, de acuerdo con un estudio realizado por la Contraloría General de la República (CGR), solamente un 36,3 \% de las instituciones públicas indican que cuentan con planes de continuidad y se observa un rezago en la implementación de medidas, en relación con la gestión financiera ante la emergencia (CGR 2020). En el caso de los sectores productivos, 
no se cuenta con evidencia de la existencia de planes de contingencia como parte de las medidas de preparación.

Se destaca la importancia de fortalecer el ámbito de la prevención para la reducción del riesgo de desastres; según la ONU (2018), por cada dólar invertido en la reducción de riesgos y la prevención se pueden ahorrar hasta USD 15 dólares en la recuperación después de un desastre. Ante lo anterior, es necesario que las organizaciones incluyan este tipo de instrumentos de planificación como planes de contingencia y estrategias de continuidad que reduzcan los efectos producidos ante la concreción de un evento disruptivo.

De acuerdo con lo anterior, es esencial promover la cultura de prevención, en donde los diferentes actores contribuyan a los procesos de reducción del riesgo. El prevenir significa una reducción significante en pérdidas económicas, parte fundamental en la sostenibilidad y crecimiento económico. Aún más importante es la reducción de pérdidas humanas que puede significar, lo que genera una sociedad que apunta hacia un desarrollo sostenible de forma integral.

La importancia de incorporar la continuidad de los servicios en los sectores productivos se establece en el Marco de Sendai 2015-2030, donde en la prioridad 3 se establece: "invertir en la reducción del riesgo de desastres para la resiliencia”, se destaca el objetivo de aumentar la resiliencia de las operaciones comerciales y la protección de los medios de vida y los bienes de producción en todas las cadenas de suministro, asegurar la continuidad de los servicios e integrar la gestión del riesgo de desastres en los modelos y prácticas comerciales (ONU 2015, 19).

Así como en la prioridad 4, que dice: "aumentar la preparación para casos de desastre a fin de dar una respuesta eficaz y reconstruir mejor en los ámbitos de la recuperación, la rehabilitación y la reconstrucción", se incluye el objetivo de asegurar la continuidad de las operaciones y la planificación, incluida la recuperación social y económica, y la prestación de servicios básicos en la fase posterior a los desastres (ONU 2015, 19).

Asimismo, en la legislación costarricense, mediante la Ley General de la Administración Pública, se establece que los servicios públicos deben regirse por el principio de continuidad: la actividad de los entes públicos deberá estar sujeta en su conjunto a los principios fundamentales del servicio público, para asegurar su continuidad, su eficiencia, su adaptación a todo cambio 
en el régimen legal o en la necesidad social que satisfacen y la igualdad en el trato de los destinatarios, usuarios o beneficiarios (CR Asamblea Legislativa 1978).

Por su parte, en la Política Nacional de Gestión del Riesgo 2016-2030 se destaca que la función pública está urgida de un enfoque de continuidad de negocios y servicio y la generación de capacidades para la planificación contingente, en donde las previsiones necesarias tengan como orientación fundamental, la continuidad de los servicios públicos y no solo la protección o recuperación de las obras, aspecto que resulta importante en un enfoque de recuperación económica y social (CR CNE 2015).

Ante lo mencionado, se evidencia la necesidad de un cambio de enfoque, pasando de la continuidad del negocio a la continuidad del servicio público, donde se refleja que son actividades realizadas por el Estado con el objetivo de satisfacer las necesidades de la ciudadanía y, por ende, su prestación de forma continua, lo que constituye una obligación para las instituciones y un derecho para la ciudadanía (CGR 2020).

En la medida en que los sectores y organizaciones públicas aseguren la prestación de sus servicios a la ciudadanía, reflejarán una mejor calidad del servicio, mayor satisfacción por parte de los beneficiarios y creación de valor público para la sociedad, al mismo tiempo que se hace uso adecuado de los recursos públicos, evitando pérdidas producto de la paralización de las actividades.

En ese sentido, se genera una necesidad por parte de los gobiernos de instrumentos normativos que permitan ser más eficientes y efectivos aplicando criterios de continuidad a sus operaciones y retomando su actividad, con miras a reactivar la economía y asegurar el cumplimiento de los lineamientos sanitarios dictados por la autoridad competente en materia de salud.

Las organizaciones, en seguimiento a dichos lineamientos, deberían comenzar a desarrollar aún más resiliencia desarrollando una agenda enfocada a reducir el impacto negativo de los eventos adversos y, por consiguiente, maximizar el efecto positivo, en la medida en que las políticas y acciones de continuidad propician una mayor probabilidad de aprovechar las oportunidades. Con lo anterior, la organización deja de invertir su tiempo en reparar y responder ante riesgos que se materializan, y avanzan a la exploración de nuevas oportunidades, aquellas que le permitan dar una mejor respuesta en 
un tiempo idóneo (Denyer 2017) y, como no decir, a nuevas oportunidades de negocio.

Seguidamente, la organización podría reconsiderar su agenda y dejar de lado la consistencia rígida de sus procesos y objetivos para aceptar mayor flexibilidad en ellos, en donde se permita de una manera sencilla la aceptación de nuevas ideas, nuevos puntos de vista y soluciones innovadoras que permitan mitigar los riesgos (Denyer 2017).

\section{Conclusiones y recomendaciones}

La región latinoamericana es propensa a diversas amenazas y sucesos disruptivos que pueden afectar el funcionamiento y operación de las organizaciones, razón por la cual, es necesario el desarrollo de estrategias que permitan continuar con las operaciones y evitar un costo mayor de recuperación.

La pandemia ocasionada por la COVID-19 implica un reto para los países en la lucha contra la propagación del virus, las medidas de confinamiento y estabilidad económica. Producto de lo anterior, es necesario la creación de documentos normativos y nuevas formas de política pública que brinden un marco de acción común y orienten las acciones del sector público y privado.

Uno de dichos documentos orientadores corresponden a los lineamientos sanitarios del Ministerio de Salud para la atención de la emergencia por la COVID-19, los cuales, además de ser una herramienta para la difusión y consulta de los acuerdos, normas, reglamentos y demás disposiciones publicadas, cuentan con una base sólida de legitimidad jurídica e institucional para su acatamiento obligatorio en todo el territorio nacional, son de aplicación general o específica, y corresponden a acciones preventivas, de mitigación y rehabilitación dictadas por el ente rector en salud (CR Ministerio de Salud 2020).

Estos documentos normativos establecen, además, que cualquier actividad económica debe contar con protocolos específicos para abordar la higiene tanto del lugar de trabajo como de las personas colaboradoras del lugar, y para la forma y condiciones como se va a brindar los servicios que prestan.

De igual forma, la normativa es aplicable a las organizaciones públicas, tanto para aquellas que brindan servicios a la ciudadanía como aquellas que 
no brindan un servicio al público pero que deben resguardar la seguridad de las personas colaboradoras, tanto si realizan trabajo en las instalaciones de la organización, como si realizan trabajo de campo, al mismo tiempo que aseguran la continuidad de sus servicios.

El documento INTE/DN-MP-S-19:2020 es importante para la ciudadanía, porque les permite conocer los protocolos que se deben aplicar en el momento de realizar sus actividades y solicitar servicios a las diferentes organizaciones, tanto públicas como privadas. De igual forma, se asegura el cumplimiento de las disposiciones sanitarias, con una rigurosidad técnica que debe contener un protocolo sanitario y que permita a los sectores implementar protocolos de una forma rápida y sencilla. En ese sentido, es esencial que desde las instancias correspondientes se fortalezca el proceso de seguimiento y monitoreo de la implementación de los protocolos, esto con el fin de contar con información clave para mejora y correcto acatamiento de las disposiciones sectoriales, así como para la evaluación de políticas públicas a nivel nacional.

Finamente, se presentan cuatro recomendaciones básicas para mejorar la capacidad de las organizaciones y adaptarse a cambios en el contexto actual de la pandemia:

- Implementar la estandarización de instrumentos de procedimiento, lo cual permite a las organizaciones atender de una manera sencilla y eficiente los requisitos que se esperan de su parte y al mismo tiempo que transmita seguridad y confianza a quienes requieren de sus servicios y a quien los regula.

- La organización debe propender a generar resiliencia, inicialmente, mediante el seguimiento de estos instrumentos estandarizados.

- Desarrollar una agenda de gestión del riesgo que bien entendida debe permitir reducir los impactos negativos de los eventos adversos y maximizar las oportunidades.

- Avanzar de procesos consistentes a procesos flexibles, que admiten cambios y modificaciones que den paso a soluciones innovadoras.

- Se destaca la importancia y necesidad de la creación de documentos normativos y nuevas formas de política pública que brinden un marco de acción común y orienten las acciones del sector público y privado ante la ocurrencia de eventos graves debido a las diversas amenazas y sucesos 
disruptivos que pueden afectar el funcionamiento y operación de las organizaciones, razón por la cual es necesario el desarrollo de estrategias que permitan continuar con las operaciones y evitar un costo mayor de recuperación.

\section{Referencias}

Asamblea General de las Naciones Unidas. 2016. Informe del grupo de trabajo intergubernamental de expertos de composición abierta sobre los indicadores y la terminología relacionados con la reducción del riesgo de desastres. Nueva York: ONU.

Bloom, David, Daniel Cadarette y Jaypee Sevilla. 2018. "Epidemias y economía: las enfermedades infecciosas nuevas y recurrentes pueden tener amplias repercusiones económicas". Finanzas y Desarrollo 55 (2): 46-49. https://bit.ly/34Upsys.

Casa Presidencial de Costa Rica. 2020. "Flujo de desarrollo, aprobación y liberación de protocolo sectorial". Video de Youtube, por Casa Presidencial de Costa Rica. Accedido diciembre de 2020. https://bit.ly/3aluoPp.

Centro de Investigación sobre la Epidemiología de los Desastres. 2017. "EM-DAT: The International Disaster Database". Bruselas: Facultad de Salud Pública de la Universidad Católica de Lovaina. Accedido octubre de 2020. https://bit.ly/2SYme7z.

Comisión Económica para América Latina y el Caribe (CEPAL). 2020. Planificación para la reducción del riesgo de desastres en el marco de la Agenda 2030 para el Desarrollo Sostenible. Santiago: CEPAL. https://bit.ly/3apoxZM.

CR. 1978. Ley General de Administración Pública. Diario Oficial La Gaceta No. 102, 30 de mayo.

CR Comisión Nacional de Prevención de Riesgos y Atención de Emergencias (CNE). 2015. Politica Nacional de Gestión del Riesgo. San José: CNE.

CR Contraloría General de la República. 2020. "Continuidad del servicio público centrado en la ciudadanía ante la emergencia". Contraloría General de la República. Accedido octubre. https://bit.ly/314H61i.

CR Presidencia de la República y Ministerio de Salud. 2020. Directriz No. 082-MPS. "Sobre los protocolos para la reactivación y continuidad de los sectores. Alcance No. 99", Diario Oficial La Gaceta No. 93, 27 de abril.

Denyer, David. 2017. "Organizational Resilience: A Summary of Academic Evidence, Business Insights and New Thinking". BSI and Cranfield School of Management. Accedido octubre de 2020. https://bit.ly/3jZ9bPa.

Gala, Ángela. 2005. "Capacidad de respuesta a emergencias epidémicas: recomendaciones para la autoevaluación nacional”. Revista Panamericana de Salud Pública 18 (2): 139-148. https://bit.ly/31aipA. 
Gómez, Liliana. 2017. "La comunicación de riesgo en salud: aspectos teóricos y metodológicos para el control de emergencias públicas sanitarias". MEDISAN 21 (12): 7017-7030. https://bit.ly/3j1FXxN.

Gonzales, Jorge, Luis Castillo, Moisés Domínguez y Víctor Ocaña. 2020. "Pandemia de la COVID-19 y las políticas de salud pública en el Perú: marzo-mayo 2020”. Revista Salud Pública 22 (2): 1-9. https://doi.org/10.15446/rsap.V22n2.87373.

González, Ignacio, Rafael Castro y Francisco del Busto. 2002. "Desastres y salud pública: un abordaje desde el marco teórico de la epidemiología”. Revista Española Salud Pública (76): 121-132. https://bit.ly/2HUcNEe.

Harrington, James. 1994. Mejoramiento de los procesos de la empresa. Bogotá: McGraw-Hill.

Instituto de Normas Técnicas de Costa Rica (INTECO). 2015. "INTE ISO 22301:2015. Seguridad de la Sociedad. Sistemas de gestión de la continuidad del negocio. Requisitos". San José: INTECO.

Jordan, Douglas, Terrence Tumpe y Barbara Jester. 2019. "La influencia más mortal: la historia completa del descubrimiento y la reconstrucción del virus de la pandemia 1928". Centros para el Control y la Prevención de Enfermedades. Accedido diciembre de 2020. http://bit.ly/3roxYQA.

Mira de Jesús, Crisalia Yunuen. 2016. "La estandarización de procesos como herramienta de mejora a la calidad de procesos administrativos". Tesis de licenciatura, Universidad Nacional Autónoma de México, Ciudad de México. https://bit.ly/3nP64vo.

Morales Nelson, Wilfredo Gálvez, Carlos Chang, Daniel Alfaro, Abel García, Marlon Ramírez, Juan Almeyda y Luis Benavente. 2008. "Emergencias y desastres: desafíos y oportunidades (De la casualidad a la causalidad)". Revista Peruana de Medicina Experimental y Salud Pública 25 (2): 237-242. https://bit.ly/3nMRNiV.

Organización de las Naciones Unidas (ONU). 2015. Marco de Sendai 2015-2030. Nueva York: ONU. http://bit.ly/39wKzdv.

Organización Panamericana de la Salud (OPS). 2005. Reglamento Sanitario Internacional. Ginebra: OPS. https://bit.ly/38tUTkX.

---. 2015. Evaluación rápida de riesgos de eventos agudos de salud pública. Washington D. C.: OPS. https://bit.ly/3k1rKCe.

Robles, José. 2020. "La psicología de emergencias ante la COVID-19: enfoque desde la prevención, detección y gestión operativa del riesgo”. Revista Clínica y Salud 31 (2): 115-118. https://doi.org/10.5093/clysa2020a17.

Sistema Económico Latinoamericano y del Caribe (SELA). 2013. La continuidad de negocios y operaciones frente a situaciones de desastre en América Latina y el Caribe. Balance y recomendaciones. Caracas: SELA. https://bit.ly/3748b8J. 